\title{
EFFECT OF COLLATAPE® COLLAGEN WOUND DRESSING ALONE AND COMBINED WITH INGENIOS $®$ SYNTHETIC BONE GRAFT ON THE SOCKET HEALING IN RABBITS Moustafa $\mathrm{H}^{1}$, Omar $\mathrm{S}^{2}$, Osman $\mathrm{S}^{3}$, Kawana $\mathrm{K}^{4}$
}

\begin{abstract}
:
Introduction: Socket preservation enhances healing and reduces bone loss during healing of extraction socket.

Objectives: To assess the effect of bio-absorbable collagen wound dressing (CollaTape ${ }^{\circledR}$; Zimmer) on the of healing of extraction sockets in rabbits when applied alone and combined with IngeniOs Beta tricalcium phosphate $(\beta$-TCP) synthetic bone particles.

Materials and methods: Sixteen rabbits were included in this study in which lower left first premolars were extracted and then divided into three groups including: Control group (4 animals) in which the sockets were left for normal healing, Collatape group (the sockets were closed with CollaTape collagen wound dressing alone) and IngeniOs $\beta$-TCP group ( 6 animals) in which the sockets were filled with synthetic bone particles and were closed with CollaTape collagen wound dressing. Euthanasia were done 2 and 6 weeks respectively. Socket healing was examined and evaluated histologically and analyzed histomorphometrically.

Results: At 2 weeks of healing, it was observed in all groups that the sockets were filled with newly formed woven bone. The percentage of newly formed bone was lowest in control group followed by Collatape group while $\beta$-TCP group showed highest values. At 6 weeks healing period, the socket entrance was sealed with compact bone and the rest of the socket was filled with trabecular bone. $\beta$-TCP group showed the highest values of the percentage of the formed bone.
\end{abstract}

Conclusions: It was confirmed that IngeniOs $\beta$-TCP enhances bone formation during socket healing and could be used for socket preservation Key Words: Socket healing, $\beta$-TCP, bone graft, Collatape

1 Department of Oral Biology, Faculty of Dentistry, Alexandria University

2 Prof, Department of Oral Biology, Faculty of Dentistry, Alexandria University

3 Prof, Department of Oral Surgery, Faculty of Dentistry, Alexandria University

4 Prof, Department of Oral Biology, Faculty of Dentistry, Alexandria University

\section{INTRODUCTION}

Millions of teeth are extracted every year. Tooth extraction is one of the most common procedures that are carried out in dental practice (1). Unfortunately the healing of dental extraction socket is a time dependent complicated process and results in pronounced resorption of alveolar process that hinder the replacement of the missing tooth.

Many attempts were attributed to assist healing and to counteract such resorption (2). Various grafting and barrier materials were used for socket preservation. Bone grafting materials are important in regenerative dentistry. They act in three different modalities involving osteogenesis, osteoinduction and osteoconduction (3). Concerning osteogenesis, the grafting material accommodates osteogenic cells that directly deposit bone. Only freshly harvested autogenous bone graft contains these osteogenic cells (4). For osteoinduction, the graft material has differentiating factors that promote differentiation of mesenchymal cells into osteoblasts. Allografts are working with this concept (5). The last is the osteoconduction in which the bone graft materials act only as a scaffold that support new bone formation.

This property is represented in Xenografts and synthetic bone grafts (6). One of the osteoconductive synthetic bone grafts is beta tri calcium phosphate $(\beta-\mathrm{TCP})$. The main disadvantage of $\beta$-TCP is its fast resorption so its degradation is not always associated with bone healing (7).

Recently, Silicated $\beta$-TCP graft material was produced. Silica was added to $\beta$-TCP in order to enhance its strength and its biologic properties so that the resorption of graft will be in co-incidence with new bone deposition (8). Researches dealing with using silicated $\beta$-TCP for socket healing are limited.

So the aim of this study is to evaluate the effect of bioabsorbable collagen wound dressing (CollaTape ${ }^{\circledR}$; Zimmer) on healing of extraction sockets in rabbits alone and combined with IngeniOs Beta tricalcium phosphate synthetic bone particles.

\section{MATERIALS AND METHODS}

The Ethical committee of Alexandria University approved the protocol of this research. Sixteen healthy male rabbits weighting $2.5 \mathrm{~kg}$ aged 14-16 weeks were used in this study. The animals were obtained from the Institute of Medical Research Alexandria University. They were housed in specially designed wire mesh bottom cages. The animals were supplied a regular balanced diet during the whole experiment period. The rabbits were divided into three groups.

Control group (4 rabbits): The sockets were left without application of any material for normal healing. CollaTape group (6 rabbits): The sockets were closed with CollaTape collagen wound dressing only. IngeniOs $\beta$-TCP group (6 rabbits): The sockets were filled with IngeniOs Beta tricalcium phosphate synthetic bone particles and were closed with CollaTape collagen wound dressing.

The animals in each group were divided equally into two subgroups each consisting of 3 animals of both CollaTape 
group and IngeniOs $\beta$-TCP group and 2 animals of control group and were scarified after 2 and 6 weeks from the start of the surgical procedure. During the surgical procedure all animals were anesthetized with general anesthesia using mixture of $13 \mathrm{mg} / \mathrm{kg}$ of body weight $2 \%$ xylazine hydrochloride and $33 \mathrm{mg} / \mathrm{kg}$ of body weight ketamine base.

In all rabbits, only the mandibular left first premolar was extracted. A mouth prop was used to obtain proper accessibility to lower left premolar. The tooth was luxated by using surgical elevators. Once luxation was performed, the tooth was pulled carefully out of the socket by lower remaining root extraction forceps. For the control group, the sockets were left without application of any materials to allow for normal healing.

For CollaTape group, a Collatape sheet was trimmed into small pieces to the size of extraction socket using surgical scissor, then it was sutured to the extraction socket by 4-0 black silk suture using the cross mattress suturing technique to achieve proper stability. For IngeniOs $\beta$-TCP group, Particles of IngeniOs $\beta$-TCP were firstly mixed with saline to be applied to the extraction socket using periosteal elevator, then Collatape absorbable collagen wound dressing was sutured to the socket using the same technique.

Post-operatively, each animal received the same course of antibiotic of Ampicillin $25 \mathrm{mg} / \mathrm{kg}$ body weight for five days after surgery every eight hours. Analgesics were given to animals in the form of Cataflam IM every eight hours for the first two days. The animals were observed daily for the first week to assess the occurrence of any signs of inflammation or infection.

Euthanasia was done at 2 and 6 weeks respectively then the mandibles were dissected out, sectioned into two halves and fixed in $10 \%$ neutral buffered formalin.

After fixation, the mandibles were decalcified in 5\% trichloroacetic acid, washed, dehydrated in ethanol and embedded in paraffin wax. Serial bucco-lingual sections of $5 \mu \mathrm{m}$ thickness were cut and stained with Hematoxylin \& Eosin.

Histomorphometric analysis using Image $\mathbf{J}$ software was done to obtain the percentage of surface area of the formed bone compared to the total surface area of the extraction socket in the two studied observation periods of socket healing (9).

Five sections of tissue from different standardized depths were used for quantification from each sample. A rectangle with standardized dimensions was drawn on the desired area to be measured using the image $\mathrm{J}$ program. The surface area of this selected region was measured by choosing Region of interest (ROI) manager, from tools from analyze and the measurement was recorded. The surface area occupied by the marrow spaces was selected using the wand tracing tool and the measurement was recorded.

The two recorded measurements were subtracted to obtain the surface area occupied only by bone, and its percentage to the total area selected was calculated. The measurements from three photographs were recorded and their mean was calculated for each of the five sections obtained, from each specimen.
Statistical analysis of the obtained data was done using ANOVA test to compare between three groups and test to compare between one group and the other two groups.

\section{RESULTS}

\section{Histological results}

Results of the first observation period (two week post operatively):

A- Control group: The apical half of the extraction socket was occupied by newly formed bone that consisted of anastomosing bone trabeculae of moderate thickness. The newly formed bony trabeculae were lined by discontinuous layer of active osteoblasts, contained moderate density of osteocytes and enclosed bone marrow spaces of limited blood supply. (Fig.1)

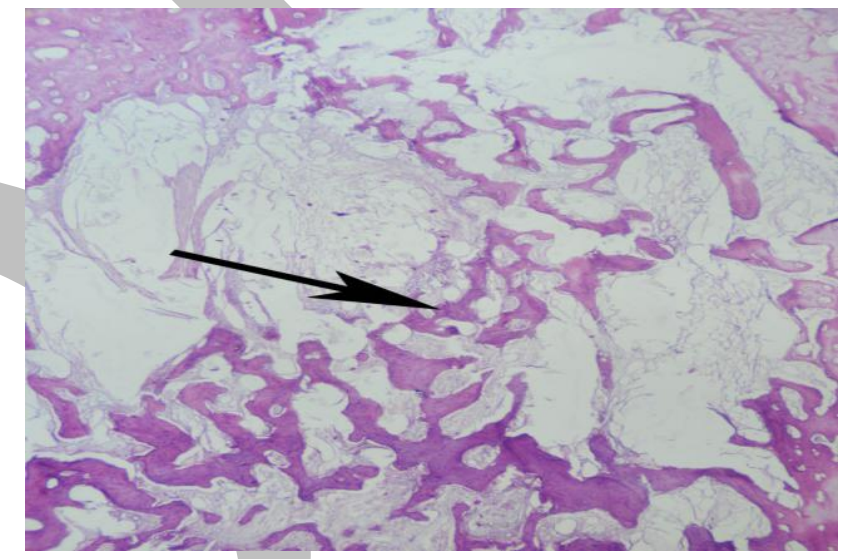

Fig.1: Light Micrograph (LM) of healing socket (Control group, 2 weeks) showing formation of bone trabeculae (arrow) of variable thickness and organization and their extension towards the center of the socket. H\&E stain original magnification X40.

B- CollaTape group: In this group, a central mass of newly formed bone was seen projecting from the lateral wall of the socket and extending towards its apical part. The coronal portion of the socket contained a mass of newly formed bone beneath Collatape collagen wound dressing. The newly formed bone trabeculae enclosed marrow spaces with rich blood supply and surrounded by dense fibrous connective tissue. They contained numerous osteocytes and lined by a continuous layer of voluminous osteoblasts. (Fig.2)

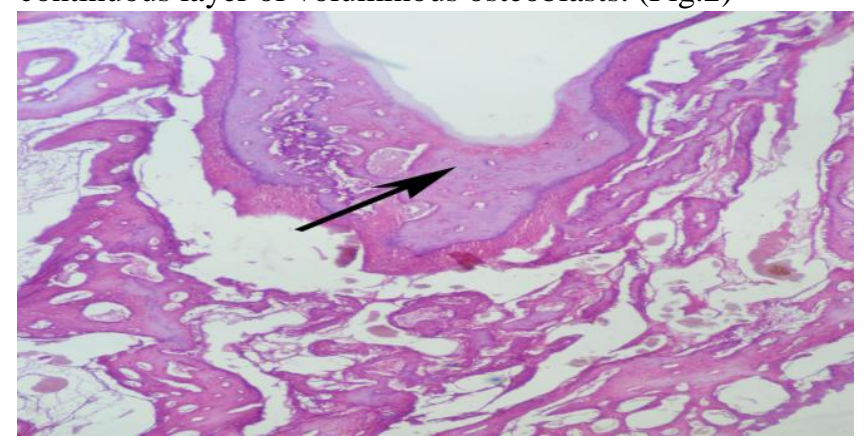

Fig.2: LM (Collatape group, 2 weeks) showing dense newly formed bone mass (arrow) extending from the lateral walls towards the center of the apical part of the healing socket. $\mathrm{H} \& \mathrm{E}$ stain original magnification X40. 
C- Ingenios $\beta$-TCP group: The apical half of the extraction socket was occupied by newly formed bone surrounded by dense fibrous connective tissue and the marginal portion of the socket harbored fibrous connective tissue. $\beta$ - TCP particles were apparently enclosed within the newly formed woven bone.

The newly formed woven bone was found at the lateral walls and at the central part of the socket surrounding $\beta$ TCP particles. The bony trabeculae contained high density of osteocytes, lined by continuous layer of active osteoblasts and enclosed bone morrow spaces containing rich blood supply. Osteoclasts were also found surrounding $\beta$-TCP particles. (Fig.3)

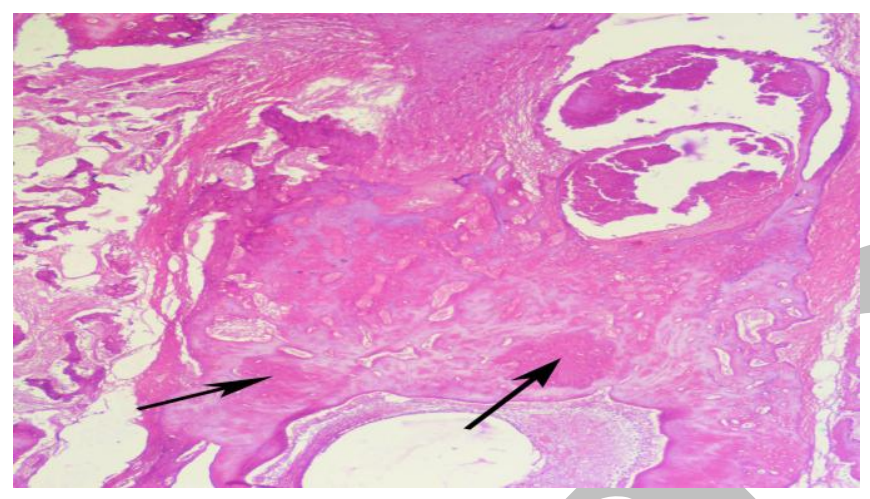

Fig.3: LM ( $\beta$-TCP group, 2weeks) showing the healing socket filled with newly formed bone trabeculae surrounding $\beta$-TCP particles (arrows). H\&E stain original magnification X40.

Results of the second observation period (six week post operatively):

A- Control group: The socket was sealed by marginal compact bone while its rest was occupied by trabecular bone surrounded by vascular spaces and fibrous tissue. An outstanding fashion of trabecular formation could be traced where the fibrous tissue modeled and mapped the shape and organization of these trabeculae in a step advancing their mineralization. (Fig.4)

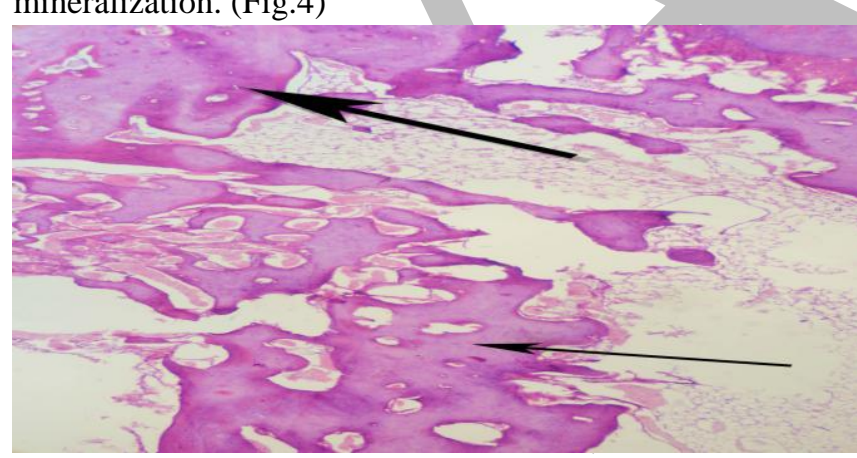

Fig.4: LM (Control group, 6weeks) showing compact bone (thick arrow) at the marginal part of the socket while the rest of the socket is filled with trabecular bone (thin arrow) enclosing vascular spaces. H\&E stain original magnification X40.

B- Collatape group: In this group, the socket was filled with mature compact bone at its coronal portion of the socket while trabecular bone was seen directed towards the apical part of the socket and surrounded by marrow spaces of rich blood supply. The compact bone was of higher density than in control group consisted of haversian systems enclosing central haversian canals. (Fig.5)

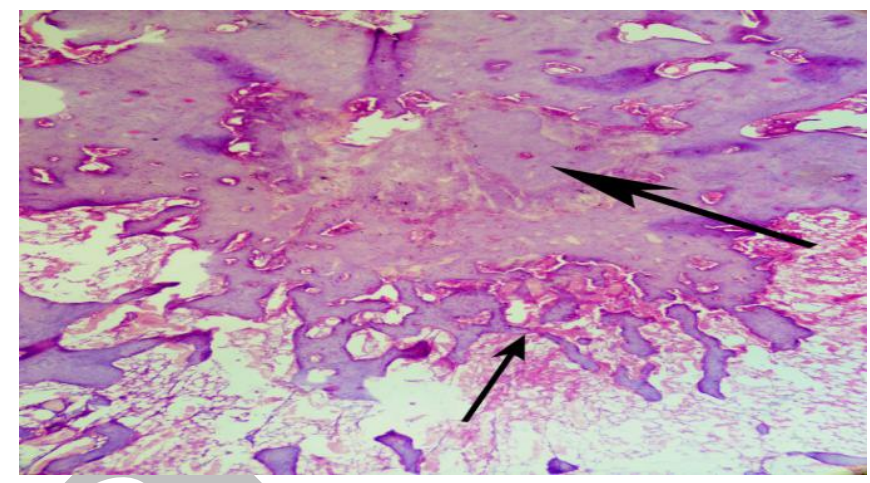

Fig.5: LM (Collatape group, 6 weeks) showing mature compact bone (thick arrow) at the coronal part of the socket with continuation of extension of the underlying trabecular bone (thin arrow) towards the apical part of the socket. H\&E stain original magnification X40.

C- IngeniOs $\boldsymbol{\beta}$-TCP group: In this group, the socket was entirely filled with dense compact bone and small areas of trabecular bone mass. The compact bone consisted of numerous haversian systems enclosing rich blood supply within their haversian canals. The compact bone was of higher density than in the previous two groups. It consisted of numerous haversian systems enclosing haversian canals. (Fig.6)

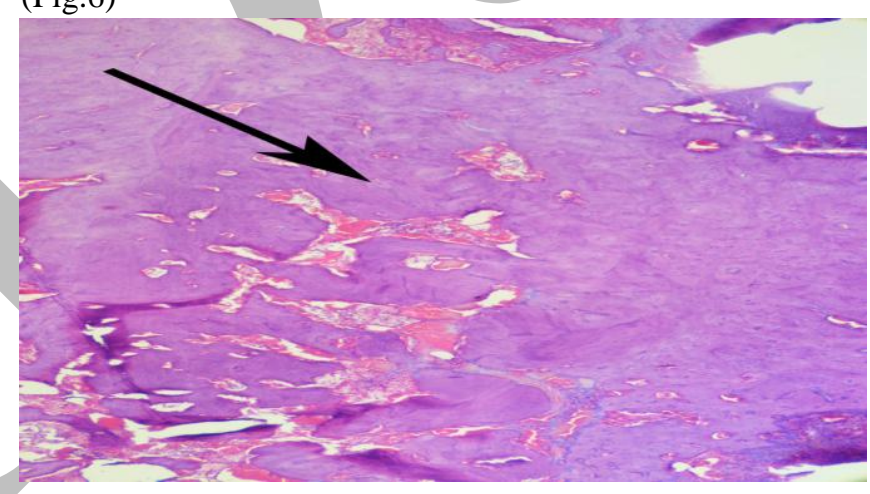

Fig.6: LM ( $\beta$-TCP group, 6weeks) showing dense compact bone mass (arrow) filling the healing socket. Note the presence of rich blood supply adjacent to the formed bone mass. $\mathrm{H} \& \mathrm{E}$ stain original magnification X40.

\section{Histomorphometric analysis}

The mean values of the percentage of the bone surface area formed in the healing socket in the control group, Collatape group and IngeniOs $\beta$-TCP group after 2 and 6 weeks from start of the experiment are demonstrated in table (1).

\begin{tabular}{|l|c|c|c|c|c|}
\hline & $\begin{array}{c}\text { Control } \\
\text { group }\end{array}$ & $\begin{array}{c}\text { Collatape } \\
\text { group }\end{array}$ & $\begin{array}{c}\boldsymbol{\beta} \text {-TCP } \\
\text { group }\end{array}$ & $\mathbf{F}$ & P \\
\hline $\begin{array}{l}\text { 2 weeks } \\
\text { Mean } \pm \text { SD }\end{array}$ & $27.77 \pm 8.88$ & $35.68 \pm 4.58$ & $38.43 \pm 12.75$ & 2.45 & 0.11 \\
\hline $\begin{array}{l}\text { 6 weeks } \\
\text { Mean } \pm \text { SD) }\end{array}$ & $\begin{array}{c}62.71 \pm 15.8 \\
2 * 1\end{array}$ & $76.06 \pm 12.86$ & $84.19 \pm 12.56$ & 4.30 & $0.029 *$ \\
\hline
\end{tabular}

Table 1. Percentage of the newly formed bone occupying the extraction socket. F: F test ANOVA *: Statistical significance $(\mathrm{P} \leq 0.05)$.

*1: Percentage of bone surface area in control group is statistically lower than in Collatape group and $\beta$-TCP group $(\mathrm{P} \leq 0.05)$. 
At the second observation period, it was noted that the control group exhibited the lowest value (27.77\%) followed by Collatape group $(35.68 \%)$ while $\beta$-TCP group showed the highest value $(38.43 \%)$. There was no statistical significance between three groups in this interval although numerical values were obtained.

For 6 weeks period of healing, the values of the mean percentage of the formed bone exhibited the lowest value $(62.71 \%)$ for control group followed by Collatape group (76.06\%) while $\beta$-TCP group showed the highest value $(84.19 \%)$. The difference in percentage of the newly formed bone in control group and the other two groups was statistically significant $(\mathrm{p}=0.029,0.026)$ respectively and there was no statistically significant difference between $\beta$ TCP group and Collatape group $(\mathrm{p}=0.19)$.

\section{DISCUSSION}

This study was performed in order to evaluate the effect of bio-absorbable collagen wound dressing CollaTape on healing of extraction sockets in rabbits alone and combined with IngeniOs Beta tricalcium phosphate synthetic bone particles.

The first mandibular premolars were extracted to study socket healing. Mandibular incisor extraction was not preferred in order not to hinder the animal ability to eat and due to their continuous eruption that might disrupt normal healing. This is supported by Kuboki et al (10) who studied the alteration in collagen crosslinking after tooth extraction in rabbits and MansoI et al (11) who studied different biomaterials in the socket healing in rabbits.

The healing of extraction socket was evaluated at two different intervals 2 and 6 respectively. The choice of such intervals could be explained as the remodeling cycle for rabbit compact bone which is about 6 weeks (12).

In the present study, the results of first observation period ( 2 weeks after post operatively) revealed the filling of socket with newly formed trabeculae with very few amount of granulation tissue in the control group. These findings are supported by Kuboki et al (10).

The transition of fibrous tissue into bone tissue occurs as osteoprogenitor cells migrate through blood vessels. They differentiate into osteoblasts that lay down newly formed bone (13).

The healing socket of Collatape group showed two striking observations which were the presence of high density of cells within collagen wound dressing and the continuity of bone formation through areas of primary osteons together with adjacent areas of new trabecular bone formation. These findings were consistent with Almazrooa et al (14) who studied the tissue response of six different collagen membranes.

Osteoconductivity of Collatape collagen wound dressing could be explained on the basis that collagen presents a rough surface geometry for osteoblast attachment. It is hydrophilic and thus favors cell adhesion. Also Fibronectin present on the surface of osteoblasts link specifically with definite regions of the collagen molecules (15). This is also supported by Donzelli et al (16) who confirmed that collagen scaffold supports mesenchymal cells distribution and differentiation.

The presence of primary osteons within formed bone mass which was noted in the second observation period indicates the active remodeling, high density and organization of the formed bone mass in this group. The rich blood supply of the healing socket in this group is considered the main promoter for active bone remodeling (17). This is explained by Twardowski (18) et al who found that type I collagen potently stimulates angiogenesis in vitro and in vivo.

For $\beta$-TCP group, the main findings in this interval were the presence of $\beta$-TCP bone graft particles incorporated in the newly formed bone mass, the newly formed bone trabeculae were lined by voluminous osteoblasts with few osteoclasts and profound blood supply within bone marrow spaces of the formed bone mass as well as around $\beta$-TCP bone graft particles. These findings are in accordance with Berberi et al (19) who evaluated a number of physical and chemical properties in a variety of granulated mineral-based biomaterials used in dentistry.

The bone graft material used in this work was silicated $\beta$ TCP bone graft. Adding silica to $\beta$-TCP has proved to enhance its osteoconductive properties. Fei et al (20) who studied the osteogenic differentiation on different bioceramic materials. The results of their study demonstrated that adding silica to $\beta$-TCP enhanced osteoblastic differentiation and bone mineralization. Silica induced osteoblastic differentiation through induction of osteoblasts to secrete bone morphogenetic protein 2 (BMP2) that stimulates osteogenic differentiation by autocrine and paracrine action. BMP2 also regulates the expression of osteogenic marker genes such as alkaline phosphatase and osteocalcin which are responsible for bone mineralization (20).

Increasing the level of porosity of $\beta$-TCP bone graft allows the blood vessels to reach the healing extraction socket and provides an environment for collagen formation that leads to deposition of apatite crystals within its micropores (21). The bone graft used in this study is IngeniOs $\beta$-TCP which has porosity up to $75 \%$ and of pore size 250-1000 $\mu \mathrm{m}$. Such large pore size explains the rich blood supply of the healing socket and the rapid rate of bone deposition which were seen in this study. This is in accordance to Galois et al 2004 (22) who studied Bone ingrowth into two porous ceramics.

Osteoclasts are essential for cell mediated resorption of $\beta$ TCP in order to be replaced with new bone, however the rapid resorption of $\beta$-TCP disrupts the healing process (21). In this study, few osteoclasts were observed in these intervals. This could be explained on the fact that the bone graft used in this study was silicated $\beta$-TCP. Adding Silica to $\beta$-TCP was found to enhance strength of $\beta$-TCP as well as its biologic response thus silicated $\beta$-TCP characterized by its lower rate of resorption to be compatible with new bone formation $(8,23)$.

The results obtained from the second observation period (6 weeks) showed that the coronal part of the socket entrance of control group was occupied by cortical bone

Alexandria Dental Journal. (2015) Vol.40 Pages:27-32 
while the rest of the socket was filled with trabecular bone surrounded by marrow spaces. This is in agreement with the findings of Kuboki et al and Cardaropoli et al (10, 13). Corticalization is an important part of socket healing in which hard tissue cap is formed in order to seal the marginal portion of the socket. The hard tissue cap is composed mainly of compact bone (24).

The presence of wide marrow spaces surrounding trabecular bone apical to hard tissue cap could be explained as there are no stresses from forces elicited during mastication so there is no demand on mineralized bone in the areas previously occupied by the tooth (24). Thus, the trabecular bone apical to hard tissue cap remodels into marrow spaces. This explains the wide marrow spaces surrounding trabecular bone.

The organization of fibrous tissue in form of bone trabeculae before mineralization was observed. This indicates the continuity of bone formation in this group as the collagen fibers take the form of bone trabeculae before the mineralization step (17).

Concerning Collatape group, the marginal portion was occupied by larger area of compact bone of rich blood supply than in control group. As a consequence Collatape could enhance bone formation and improve the quality of the formed bone. This is in agreement with Kim (25) et al who observed that formed mature bone was found eight weeks after implantation of collagen wound dressing in comparison with untreated animals.

The presence of wide marrow space surrounding trabecular bone apical to hard tissue cap could be due to the rapid degeneration of Collatape collagen wound dressing (45 weeks) so there was no longer stimulation of new bone formation. This is supported by Donzelli et al (16) who confirmed that the rapid degeneration of collagen membranes might be a limitation for their use in bone regeneration.

In $\beta$-TCP group, the coronal portion of the socket was filled with compact bone of higher density and vascularity than in control group and Collatape group. The trabecular bone formed apical to the marginal cap was of higher thickness than in the previous two groups and were surrounded by small marrow spaces. These observations are in agreement with Brkovic et al and Jensen et al $(26,27)$. On the other hand Araujo et al (28) showed that use of the bTCP graft might in fact have retarded bone formation in the animal model used.

Such observations confirmed that IngeniOs $\beta$-TCP bone graft could effectively enhance bone formation and reduce the amount of bone resorption associated with bone remodeling.

\section{CONCLUSIONS}

1- Application of Collatape alone to the healing socket improved the early phases of bone healing, however, it did not provide long term ridge preservation.

2- Combination of Collatape and IngeniOs $\beta$-TCP could be used for socket preservation. IngeniOs $\beta$-TCP has osteoconductive properties and its resorption is compatible with new bone formation. Increasing porosity of the $\beta$-TCP graft material enhances blood supply of the healing socket and accelerates the rate of bone deposition.

\section{STATEMENT OF CONFLICT OF INTEREST}

The authors declare that they have no conflicts of interest

\section{REFERENCES}

1. Botticelli D, Berglundh T, Lindhe J. Hard-tissue alterations following immediate implant placement in extraction sites. Journal of clinical periodontology. 2004;31(10):820-8.

2. Pagni G, Pellegrini G, Giannobile WV, Rasperini G. Postextraction alveolar ridge preservation: Biological basis and treatments. International journal of dentistry. 2012;2012:1-13.

3. Dinopoulos $\mathrm{H}$, Dimitriou $\mathrm{R}$, Giannoudis PV. RETRACTED: Bone graft substitutes: What are the options? the surgeon. 2012;10(4):230-9.

4. Sándor G, Lam DK, Ylikontiola LP, Kainulainen VT, Oikarinen KS, Clokie CM. Autogenous bone harvesting techniques. Oral and Maxillofacial Surgery. 2010;23:383.

5. Minichetti JC, D'Amore JC, Hong AY, Cleveland DB. Human histologic analysis of mineralized bone allograft (Puros) placement before implant surgery. Journal of Oral Implantology. 2004;30(2):74-82.

6. Bohner M. Resorbable biomaterials as bone graft substitutes. Materials Today. 2010;13(1):24-30.

7. Kamitakahara M, Ohtsuki C, Miyazaki T. Review paper: behavior of ceramic biomaterials derived from tricalcium phosphate in physiological condition. Journal of biomaterials applications. 2008;23(3):197-212.

8. Bohner M. Silicon-substituted calcium phosphates-a critical view. Biomaterials. 2009;30(32):6403-6.

9. Yugoshi L, Sala M, Brentegani L, Carvalho $\mathrm{T}$. Histometric study of socket healing after tooth extraction in rats treated with diclofenac. Brazilian dental journal. 2002;13(2):92-6.

10. Kuboki Y, Hashimoto F, Ishibashi K. Time-dependent changes of collagen crosslinks in the socket after tooth extraction in rabbits. Journal of Dental Research. 1988;67(6):944-8.

11. MansoI J, MourãoII C, PinheiroIII F, Luiz M, FerreiraIV P, Schanaider A. Molars extraction for bone graft study in rabbits. Acta Cirúrgica Brasileira. 2011;26(2):66-9.

12. Roberts WE, Smith RK, Zilberman Y, Mozsary PG, Smith RS. Osseous adaptation to continuous loading of rigid endosseous implants. American journal of orthodontics. 1984;86(2):95-111.

13. Cardaropoli G, Araujo M, Lindhe J. Dynamics of bone tissue formation in tooth extraction sites. Journal of clinical periodontology. 2003;30(9):809-18.

14. Almazrooa SA, Noonan V, Woo S-B. Resorbable collagen membranes: histopathologic features. Oral surgery, oral medicine, oral pathology and oral radiology. 2014;118(2):236-40.

15. Jain S, Kaur H, Pandav G, Dewan A, Saxena D. Collagen: Basis of Life. Universal Research Journal of Dentistry. 2014;4(1):1-9. 
16. Donzelli E, Salvadè A, Mimo $\mathrm{P}$, Viganò $\mathrm{M}$, Morrone $\mathrm{M}$, Papagna R, et al. Mesenchymal stem cells cultured on a collagen scaffold: In vitro osteogenic differentiation. Archives of oral biology. 2007;52(1):64-73.

17. Hadjidakis DJ, Androulakis II. Bone remodeling. Annals of the New York Academy of Sciences. 2006;1092(1):385-96. 18. Twardowski T, Fertala A, Orgel J, San Antonio J. Type I collagen and collagen mimetics as angiogenesis promoting superpolymers. Current pharmaceutical design. 2007;13(35):3608-21.

19. Berberi A, Samarani A, Nader N, Noujeim Z, Dagher M, Kanj W, et al. Physicochemical Characteristics of Bone Substitutes Used in Oral Surgery in Comparison to Autogenous Bone. BioMed research international. 2014;2014:1-9.

20. Fei L, Wang C, Xue Y, Lin K, Chang J, Sun J. Osteogenic differentiation of osteoblasts induced by calcium silicate and calcium silicate/ $\beta$-tricalcium phosphate composite bioceramics. Journal of Biomedical Materials Research Part B: Applied Biomaterials. 2012;100(5):1237-44. 21. Chazono M, Tanaka T, Kitasato S, Kikuchi T, Marumo $\mathrm{K}$. Electron microscopic study on bone formation and bioresorption after implantation of $\beta$-tricalcium phosphate in rabbit models. Journal of Orthopaedic Science. 2008;13(6):550-5.

22. Galois L, Mainard D. Bone ingrowth into two porous ceramics with different pore sizes: an experimental study. Acta Orthop Belg. 2004;70(6):598-603.

23. Hing KA, Wilson LF, Buckland T. Comparative performance of three ceramic bone graft substitutes. The Spine Journal. 2007;7(4):475-90.

24. Araújo MG, Silva CO, Misawa M, Sukekava F. Alveolar socket healing: what can we learn? Periodontology 2000. 2015;68(1):122-34.

25. Kim JH, Kim CH, Kim KW. Bone healing capacity of the collagen bone filler (TERUPLUG (R)) and rhBMP-2 in the rabbit cranium defect. Journal of the Korean Association of Oral and Maxillofacial Surgeons. 2008;34(2):119-30.

26. Brkovic B, Prasad HS, Konandreas G, Milan R, Antunovic D, Sándor G, et al. Simple preservation of a maxillary extraction socket using beta-tricalcium phosphate with type I collagen: preliminary clinical and histomorphometric observations. J Can Dent Assoc. 2008;74(6):523-8.

27. Jensen SS, Broggini N, Hjørting-Hansen E, Schenk R, Buser D. Bone healing and graft resorption of autograft, anorganic bovine bone and $\beta$-tricalcium phosphate. A histologic and histomorphometric study in the mandibles of minipigs. Clinical oral implants research. 2006;17(3):237-43.

28. Araújo $\mathrm{MG}$, Liljenberg $\mathrm{B}$, Lindhe J. $\beta$-tricalcium phosphate in the early phase of socket healing: an experimental study in the dog. Clinical oral implants research. 2010;21(4):445-54. 